\title{
Characterization of gaseous emissions and ashes from the combustion of furniture waste
}

\section{Ana Isabel Moreno}

anaisabel.moreno@ua.es

\section{Rafael Font}

Juan A. Conesa

Department of Chemical Engineering, University of Alicante, P.O. Box 99, E-03080 Alicante, Spain

${ }^{*}$ Corresponding author.

\section{Abstract}

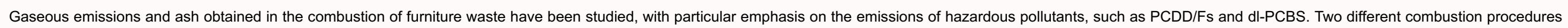

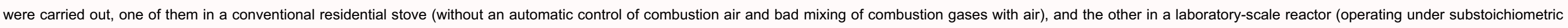
conditions).

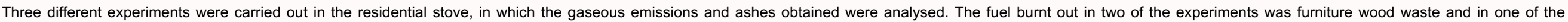

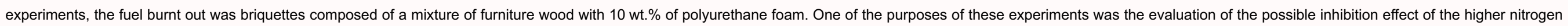

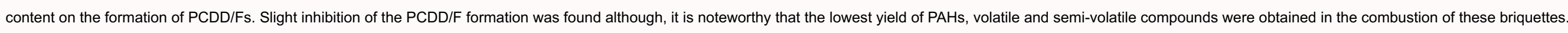

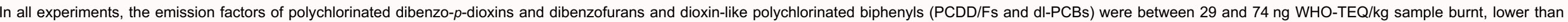
that obtained by other authors in the burning of pine needles and cones.

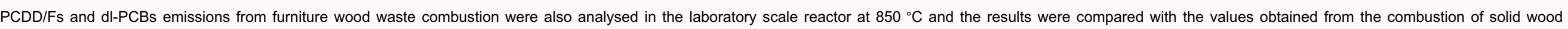

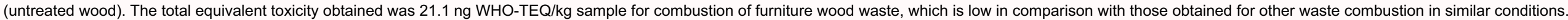

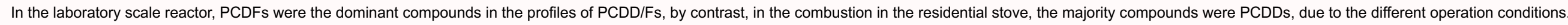

Keywords: Combustion; Furniture waste; Nitrogenated compounds; PCDD; Fs; PCBs; Organic pollutants

\section{Introduction}

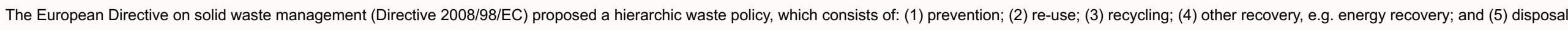

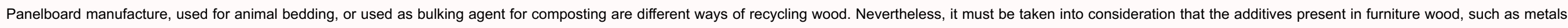

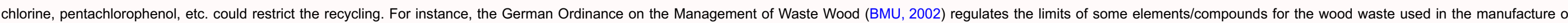
panelboard; but treated wood usually exceeds these limits. Therefore, combustion for energy recovery can be an appropriate management method for furniture wood waste, avoiding its disposal in landfills.

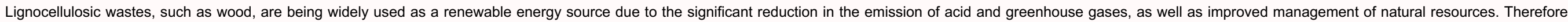
energy recovery is a possible way to manage with these wood wastes, because its use improves the management of environmental resources and wastes.

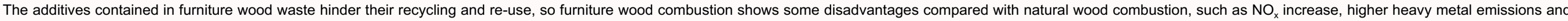
increase of PCDD/Fs, dioxin-like compounds and other organic compounds (Lavric et al., 2004).

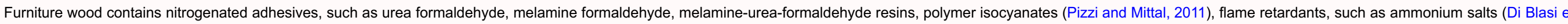




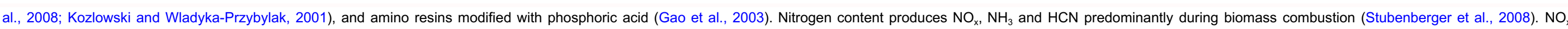

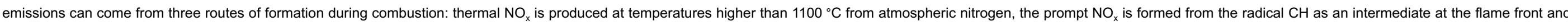
the fuel- $\mathrm{NO}_{x}$ is formed from nitrogen of the fuel (Werther et al., 2000). Nevertheless, at temperatures lower than $1000{ }^{\circ} \mathrm{C}, \mathrm{NO}_{x}$ is expected to be produced mainly from the nitrogen of the fuel (Roy and Corscadden, 2012).

Not only heavy metal emissions (particles, volatile halides, etc.) are harmful, but also the pollutant retention in the ash, which restricts its use for disposal in agricultural soil (Obernberger et al., 1997).

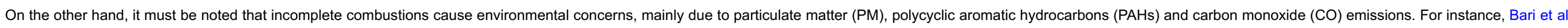

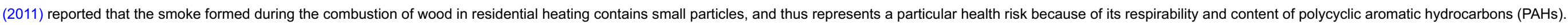

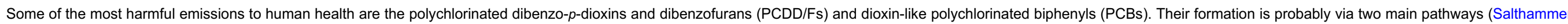

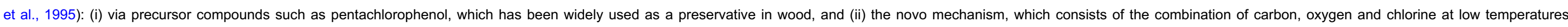
(between 200 and $400^{\circ} \mathrm{C}$ ) and catalyzed by a metal, mainly $\mathrm{Cu}$.

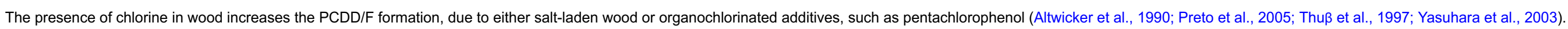

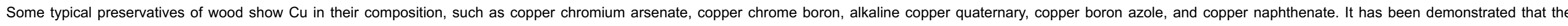

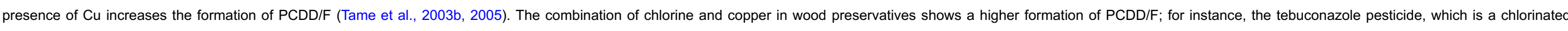
compound, present in copper boron azole preservative provides chlorinated precursors for PCDD/F formation and Cu as a catalyst (Tame et al., 2005).

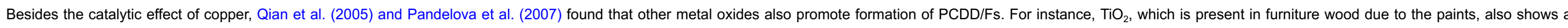
promotion effect on PCDD/F formation, although, the strongest catalytic effect was observed with CuO.

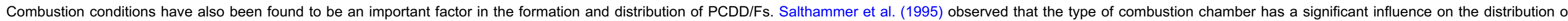

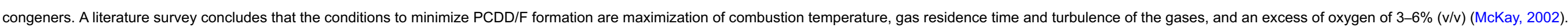

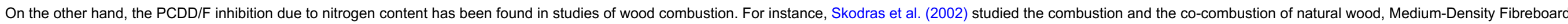

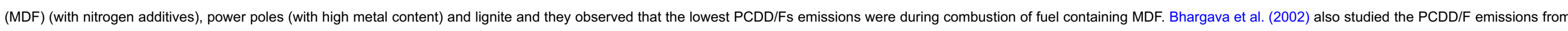
combustions of chipboard and MDF (with nitrogen additives) in a cone calorimeter. They found toxic equivalencies of 20.5 and $15.4 \mathrm{ng}$ TEQ/kg sample for chipboard and MDF, respectively.

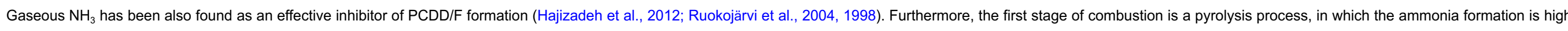
if the nitrogen content of the fuel is significant (Moreno and Font, 2015).

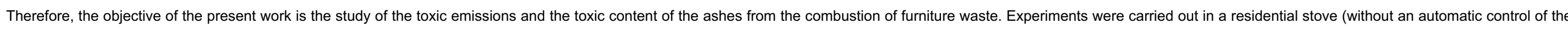

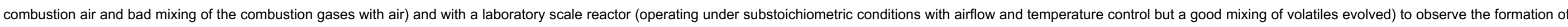
pollutants under these conditions.

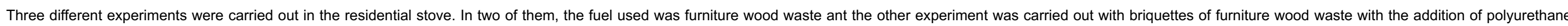

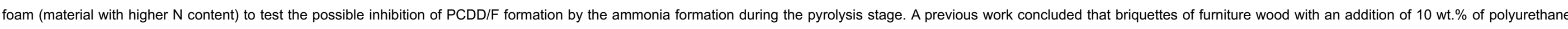
foam show good quality (durability and density of the briquettes) (Moreno et al., 2016). For this reason, this combustion experiment was carried out with briquettes of furniture wood with 10 wt\% of polyurethane foam.

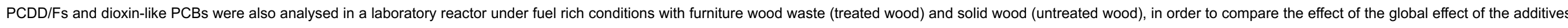

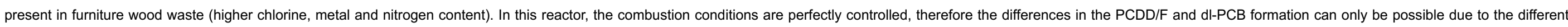
composition of the wood studied. These set-ups were carried out under substoichiometric conditions to promote the formation of products of incomplete combustion.

\section{Experimental section}

\subsection{Characteristics of the materials}




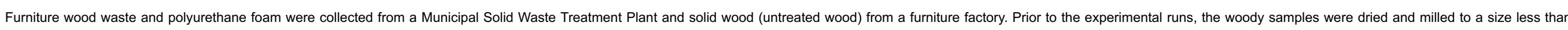
$0.2 \mathrm{~mm}$ to obtain representative samples for the further characterization of the samples and for the experimental runs in the laboratory-scale reactor.

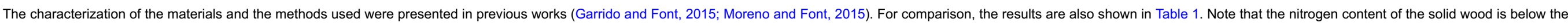
limit of detection. For some elements, the standard deviations are high due to the fact that their concentrations are low and close to the detection limit.

Table 1 Characterization of the materials used.

\begin{tabular}{|c|c|c|c|}
\hline & Furniture wood waste (Moreno and Font, 2015) & Solid wood (Moreno and Font, 2015) & Polyurethane foam (Garrido and Font, 2015) \\
\hline NCV, MJ/kg (d.b.) & $15.8 \pm 0.1$ & $19.5 \pm 0.5$ & 24.2 \\
\hline Ash content, wt\% (d.b.) & $1.8 \pm 0.1$ & $0.3 \pm 0.1$ & 5.5 \\
\hline C, wt\% (d.b.) & $47.9 \pm 0.03$ & $52.7 \pm 0.1$ & 57.8 \\
\hline $\mathrm{H}, \mathrm{wt} \%$ (d.b.) & $6.0 \pm 0.1$ & $6.2 \pm 0.1$ & 7.4 \\
\hline $\mathrm{N}, \mathrm{wt} \%$ (d.b.) & $2.86 \pm 0.58$ & $<0.01$ & 5.95 \\
\hline O, wt\% (d.b.) & $41.4 \pm 0.6$ & $40.8 \pm 0.1$ & 23.4 \\
\hline \multicolumn{4}{|c|}{ Others elements, $\mathrm{mg} / \mathrm{kg}$ (d.b.) } \\
\hline & \multicolumn{2}{|l|}{ ICP analysis } & XRF analysis \\
\hline s & $540 \pm 130$ & $70 \pm 50$ & 130 \\
\hline $\mathrm{Cl}$ & $630 \pm 70$ & $160 \pm 80$ & 390 \\
\hline Al & $480 \pm 10$ & $30 \pm 20$ & 80 \\
\hline $\mathrm{Ca}$ & $1590 \pm 240$ & $260 \pm 50$ & $>20,000$ \\
\hline $\mathrm{Fe}$ & $280 \pm 30$ & $25 \pm 4$ & 250 \\
\hline $\mathrm{Mg}$ & $320 \pm 80$ & $100 \pm 30$ & 100 \\
\hline$P$ & $100 \pm 20$ & $20 \pm 10$ & 20 \\
\hline K & $400 \pm 150$ & $160 \pm 90$ & n.d. \\
\hline $\mathrm{Si}$ & $2150 \pm 140$ & $109 \pm 3$ & 1050 \\
\hline $\mathrm{Na}$ & $300 \pm 120$ & $20 \pm 10$ & 270 \\
\hline $\mathrm{Ti}$ & $1600 \pm 200$ & $2.1 \pm 0.8$ & n.d. \\
\hline As & $0.4 \pm 0.03$ & $0.011 \pm 0.004$ & n.d. \\
\hline $\mathrm{Cd}$ & $1.0 \pm 0.1$ & $0.1 \pm 0.02$ & n.d. \\
\hline Co & $1.1 \pm 0.1$ & $0.051 \pm 0.004$ & n.d. \\
\hline $\mathrm{Cr}$ & $6.7 \pm 1.4$ & $2.0 \pm 1.3$ & n.d. \\
\hline $\mathrm{Cu}$ & $6.4 \pm 0.7$ & $1.4 \pm 1.4$ & n.d. \\
\hline $\mathrm{Hg}$ & $0.03 \pm 0.04$ & $0.006 \pm 0.003$ & n.d. \\
\hline $\mathrm{Mn}$ & $40.0 \pm 3.6$ & $46.8 \pm 7.1$ & n.d. \\
\hline Mo & $0.34 \pm 0.03$ & $0.02 \pm 0.02$ & n.d. \\
\hline
\end{tabular}




\begin{tabular}{|l|l|l|l|l|}
\hline $\mathrm{Ni}$ & $1.2 \pm 0.3$ & $0.04 \pm 0.07$ & n.d. \\
\hline $\mathrm{Pb}$ & $6.3 \pm 3.6$ & $0.04 \pm 0.06$ & $0.01 \pm 0.01$ \\
\hline $\mathrm{Sb}$ & $2.0 \pm 0.4$ & $0.052 \pm 0.002$ & n.d. \\
\hline $\mathrm{V}$ & $0.85 \pm 0.04$ & $7.7 \pm 0.9$ & n.d. \\
\hline $\mathrm{Zn}$ & $69.4 \pm 2.0$ & $0.02 \pm 0.04$ & n.a. \\
\hline $\mathrm{Br}$ & $8.8 \pm 0.8$ & \\
\hline
\end{tabular}

n.a.: not analysed.

n.d.: not detected ( $<10 \mathrm{mg} / \mathrm{kg}$ in XRF analysis).

Furniture wood waste shows high nitrogen content although the highest $\mathrm{N}$ content is in the polyurethane foam. Therefore, the briquettes used in this work (with $10 \mathrm{wt} . \%$ of polyurethane foam) show a high nitrogen content (3.17 wt.\%).

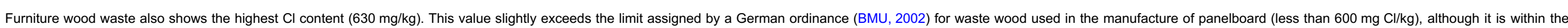
European limits proposed for non-industrial briquettes (less than $1000 \mathrm{mg} \mathrm{Cl} / \mathrm{kg}$ ) (CEN, 2010). Therefore, recovery of energy with furniture wood waste is the best option for its management.

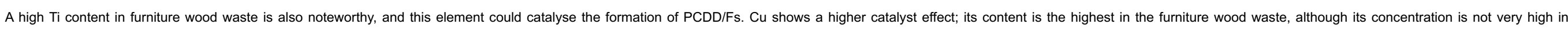
comparison with the Ti content.

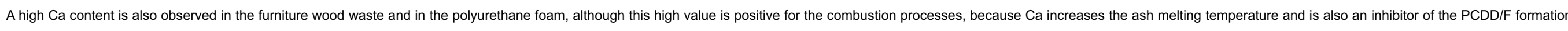
(Qian et al., 2005).

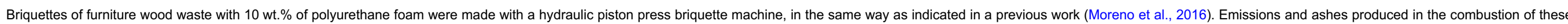
briquettes were also analysed.

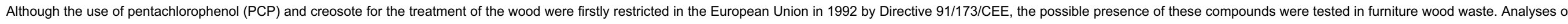

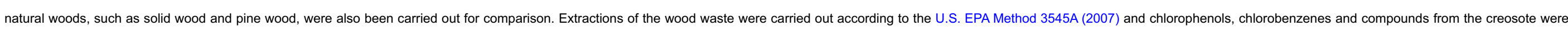
analysed according to U.S. EPA 8270D (EPA, 2014). Chlorobenzenes were not detected in any of the samples analysed. The results obtained are shown in Table 2.

Table 2 Chlorophenols, PAHs and other organic compounds in furniture wood waste and comparison with other natural woods.

\begin{tabular}{|c|c|c|c|}
\hline & Furniture wood waste $(n=2)$ & Solid wood & Pine wood \\
\hline \multicolumn{4}{|c|}{ Chlorophenols ( $\mu g / k g$ dry basis) } \\
\hline MONO- & $2.6 \pm 1.4$ & nd & nd \\
\hline DI- & $15.5 \pm 5.8$ & 0.8 & 0.6 \\
\hline TRI- & $11.1 \pm 4.5$ & 0.1 & 0.1 \\
\hline TETRA- & $58.3 \pm 25.6$ & 0.6 & 0.4 \\
\hline PENTA- & $524.4 \pm 211.1$ & 5.9 & 0.1 \\
\hline Total & $611.9 \pm 248.4$ & 7.4 & 1.0 \\
\hline \multicolumn{4}{|c|}{ PAHs ( $\mu g / k g$ dry basis) } \\
\hline Naphthalene & $84 \pm 13$ & nd & nd \\
\hline Acenaphthylene & nd & nd & nd \\
\hline
\end{tabular}




\begin{tabular}{|c|c|c|c|}
\hline Acenaphthene & $11 \pm 2$ & nd & nd \\
\hline Fluorene & $14 \pm 2$ & nd & nd \\
\hline Phenanthrene & $118 \pm 1$ & 11 & nd \\
\hline Anthracene & $16 \pm 4$ & nd & nd \\
\hline Fluoranthene & $121 \pm 3$ & 8 & nd \\
\hline Pyrene & $173 \pm 17$ & nd & nd \\
\hline Benzo(a)anthracene & $66 \pm 42$ & nd & nd \\
\hline Chrysene & $47 \pm 7$ & nd & nd \\
\hline Benzo(b)fluoranthene & $13 \pm 19$ & nd & nd \\
\hline Benzo(k)fluoranthene & $19 \pm 1$ & nd & nd \\
\hline Benzo(a)pyrene & nd & nd & nd \\
\hline Indeno(1,2,3-cd)pyrene & nd & nd & nd \\
\hline Dibenz(a,h)anthracene & nd & nd & nd \\
\hline Benzo(g,h,i)perylene & nd & nd & nd \\
\hline Total & 645 & 19 & nd \\
\hline \multicolumn{4}{|l|}{ Other organic compounds ( $\mu \mathrm{g} / \mathrm{kg}$ dry basis) } \\
\hline Pyridine, 2-methyl & $301 \pm 206$ & nd & nd \\
\hline Ethylbenzene & $159 \pm 193$ & 23 & nd \\
\hline Xylenes & $82 \pm 76$ & 17 & nd \\
\hline Cymene & $755 \pm 138$ & 901 & 151 \\
\hline Phenol & $740 \pm 156$ & nd & nd \\
\hline Cresol & $309 \pm 25$ & 202 & 65 \\
\hline Naphthalene, 1,6-dimethyl-4-(1-methylethyl)- & $136 \pm 40$ & nd & nd \\
\hline
\end{tabular}

nd: values less than limit of detection (LOD $\sim 0.1 \mu \mathrm{g} / \mathrm{kg}$ for chlorophenols, $\sim 3 \mu \mathrm{g} / \mathrm{kg}$ for PAHs and other organic compounds).

${ }^{a}$ Duplicate analysis.

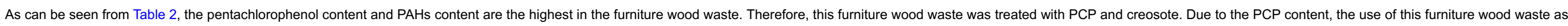
a bulking agent in composting processes is not recommended, because during composting, the PCP present in the wood could react and produce PCDD/Fs (Gómez-Rico et al., 2007; Muñoz et al., 2010, 2014).

\subsection{Experimental procedure}

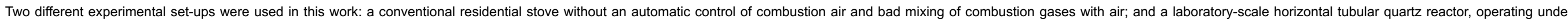

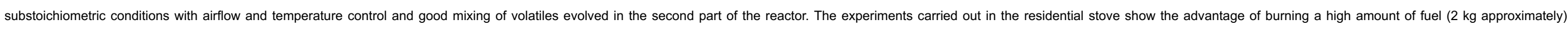
whereas, in the laboratory scale reactor, the amount of sample that is burnt is small (between 1 and $2 \mathrm{~g}$ for PCDD/F and PCB analysis).

\subsubsection{Residential Stovestove}




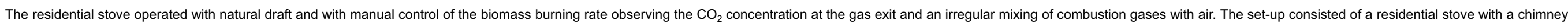
stack, and the emissions evolved were sampled (SKMM5HS, Apex Instruments, USA) according to the U.S. EPA Method 0023A (Fig. 1). Temperature was measured regularly with a temperature probe at the centre of the stove.

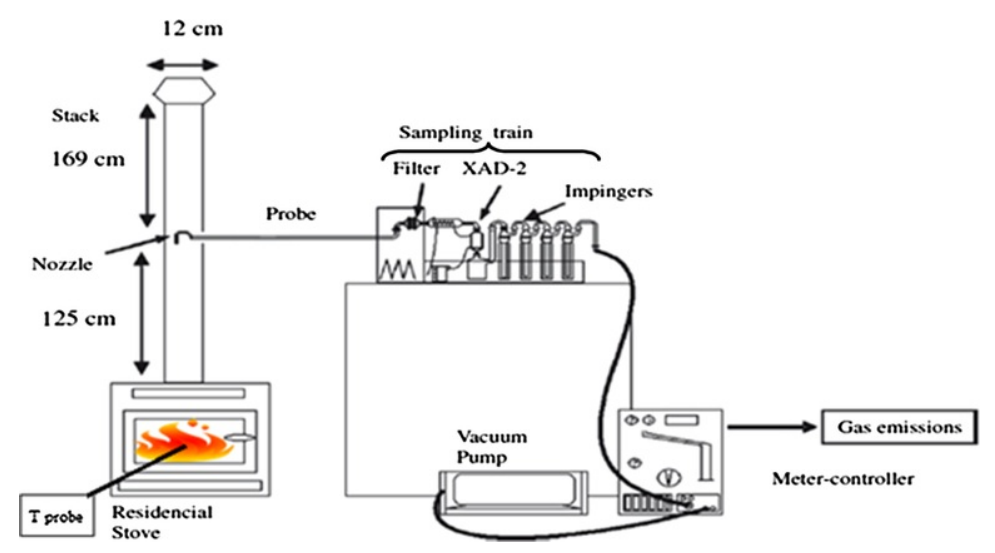

Fig. 1 Scheme of the experimental set-up (residential stove and sampling train).

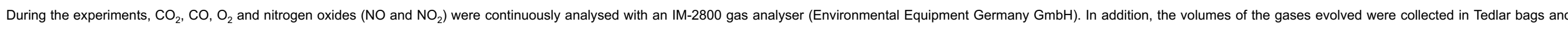
they were analysed by gas chromatography using a TCD and FID detector and by a GC-MS with a DB-624 capillary column $(30 \mathrm{~m} \times 0.25 \mathrm{~mm} \times 1.4 \mu \mathrm{m})$.

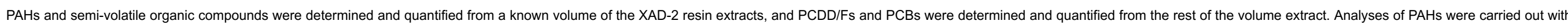

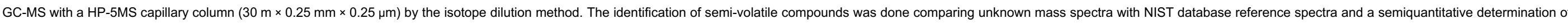

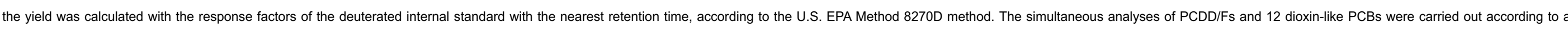

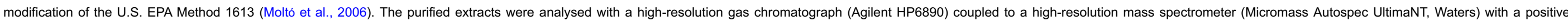
electron impact (El+) source.

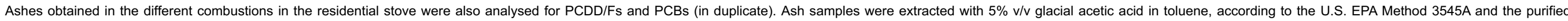

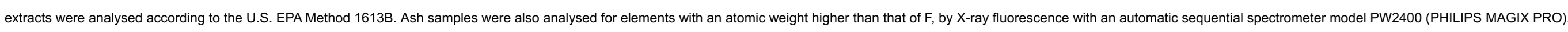

Blanks of the experiments and the equipment used were also carried out prior to the corresponding runs.

\subsubsection{Laboratory reactor}

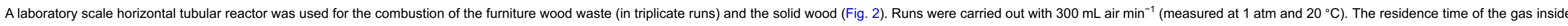

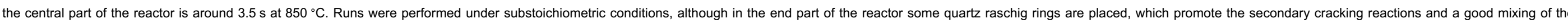

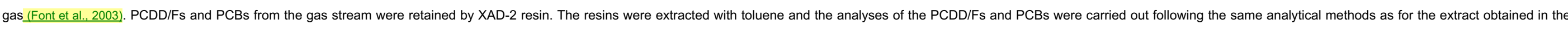
residential stove runs.

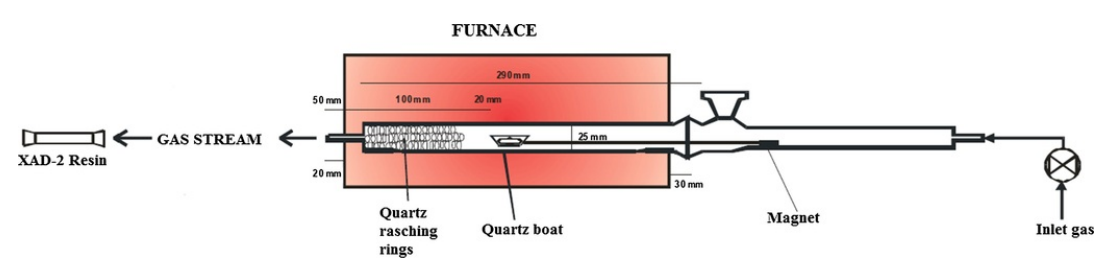

Fig. 2 Scheme of the laboratory scale reactor. 


\section{Results and discussion}

Table 3 shows the operating conditions, volume percentage of the carbon oxides and oxygen, nitrogen oxides and the yields of hydrocarbons and 16 priority PAHs generated in the residential stove experiments.

Table 3 Volatile gases and 16 priority PAHs evolved in residential stove combustion

\begin{tabular}{|c|c|c|c|}
\hline & Furniture wood waste (I) & Furniture wood waste (II) & Briquettes of furniture wood and $10 \mathrm{wt} . \%$ of foam \\
\hline \multicolumn{4}{|l|}{ Operating conditions } \\
\hline $\mathrm{Nm}^{3}$ dry gas/kg burnt & 41 & 37 & 24 \\
\hline Residence time (s) & $\sim 3.5$ & $\sim 3.5$ & $\sim 3.6$ \\
\hline Furnace temperature $\left({ }^{\circ} \mathrm{C}\right)$ & $420-780$ & $320-740$ & $450-750$ \\
\hline \multicolumn{4}{|l|}{ Carbon oxides } \\
\hline $\mathrm{CO}_{2}(\mathrm{~g} / \mathrm{kg}$ sample burned) & $1650 \pm 530$ & $1663 \pm 832$ & $1649 \pm 768$ \\
\hline CO (g/kg sample burnt) & $42 \pm 12$ & $46 \pm 19$ & $40 \pm 15$ \\
\hline Ratio $\mathrm{CO} /\left(\mathrm{CO}_{2}+\mathrm{CO}\right)$ & $0.025 \pm 0.014$ & $0.035 \pm 0.023$ & $0.030 \pm 0.018$ \\
\hline \multicolumn{4}{|l|}{ Oxygen (\% vol.) } \\
\hline $\mathrm{O}_{2}$ & $19.0 \pm 0.6$ & $18.6 \pm 1.2$ & $17.6 \pm 1.7$ \\
\hline \multicolumn{4}{|c|}{ Nitrogen oxides (g/kg sample burnt) } \\
\hline NO & $1.77 \pm 0.45$ & $2.42 \pm 0.91$ & $4.43 \pm 2.22$ \\
\hline $\mathrm{NO}_{2}$ & $<0.05$ & $<0.05$ & $<0.05$ \\
\hline \multicolumn{4}{|l|}{ Volatiles (mg/kg sample burnt) } \\
\hline Methane & 6030 & 1750 & 330 \\
\hline Ethane & 820 & 160 & 30 \\
\hline Ethene & 1890 & 350 & 220 \\
\hline Propane & nd & 20 & 30 \\
\hline Propene & 480 & 190 & 340 \\
\hline Ethyne & 560 & 60 & 50 \\
\hline Propyne & 90 & 90 & 230 \\
\hline Toluene & 14,130 & 14,050 & 1620 \\
\hline Total volatiles (mg/kg sample) & 24,000 & 16,670 & 2850 \\
\hline \multicolumn{4}{|c|}{ nd: value less than LOD $10 \mathrm{mg} / \mathrm{kg}$} \\
\hline \multicolumn{4}{|l|}{16 priority PAHs (mg/kg sample) } \\
\hline Naphthalene & 19.8 & 39.2 & 2.8 \\
\hline Acenaphthylene & 6.4 & 11.7 & 1.5 \\
\hline
\end{tabular}




\begin{tabular}{|l|l|l|l|}
\hline Acenaphthene & 0.4 & 0.7 & 0.1 \\
\hline Fluorene & 1.4 & 8.9 & 0.5 \\
\hline Phenanthrene & 3.4 & 1.0 & 1.3 \\
\hline Anthracene & 0.8 & 2.4 & 0.3 \\
\hline Fluoranthene & 1.2 & 2.0 & 0.3 \\
\hline Pyrene & 1.4 & 0.5 & 0.3 \\
\hline Benzo[a]anthracene & 0.5 & 0.6 & 0.1 \\
\hline Chrysene & 0.3 & 0.4 & 0.1 \\
\hline Benzo[b]fluoranthene & 0.2 & 0.4 & 0.1 \\
\hline Benzo[k]fluoranthene & 0.2 & 0.3 & \\
\hline Benzo[a]pyrene & 0.2 & 0.2 & nd \\
\hline Indeno[1,2,3-cd]pyrene & 0.1 & 0.2 & nd \\
\hline Dibenz[a,h]anthracene & nd & $\mathbf{7 1}$ & nd \\
\hline Benzo[g,h,i]perylene & 0.1 & $\mathbf{7 2 9}$ & nd \\
\hline Total PAHs & $\mathbf{3 6}$ & $\mathbf{8 0 7}$ & nd \\
\hline Total semi-volatile compounds & & $\mathbf{8}$ \\
\hline
\end{tabular}

nd: value less than $L O D \sim 0.1 \mathrm{mg} / \mathrm{kg}$

The operating conditions are those which are obtained in combustion in the stove without automatic control, the percentage of oxygen was the only variable that was controlled manually $\left(\right.$ around values of $18 \% \mathrm{O}_{2}$ ).

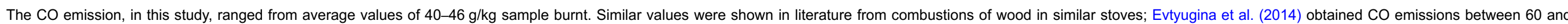
$120 \mathrm{~g} / \mathrm{kg}$, and Tissari et al. (2007) found CO emissions between 28 and $120 \mathrm{~g} / \mathrm{kg}$. In large industrial combustors, CO emissions are lower (Williams et al., 2012).

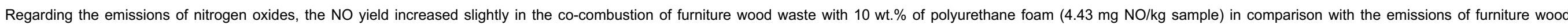
combustion (without foam; 1.77 and $2.42 \mathrm{mg} \mathrm{NO} / \mathrm{kg}$ sample). This increase is due to the higher $\mathrm{N}$ content in the briquettes used.

On the other hand, the yields of volatile compounds obtained from the combustion of the briquettes are lower than the other two experiments burning furniture wastes without polyurethane foam.

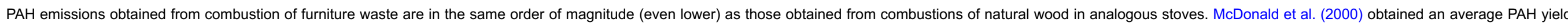
from wood stoves of $75 \mathrm{mg} / \mathrm{kg}$. Tissari et al. (2007) found $164 \mathrm{mg} \mathrm{PAH} / \mathrm{kg}$ wood, from a sauna stove combustion.

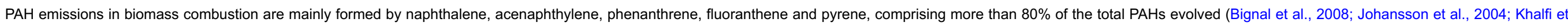
al., 2000; Reisen et al., 2014); the results shown, even for combustion of briquettes with $10 \mathrm{wt}$ \% of polyurethane foam, are in agreement with this trend.

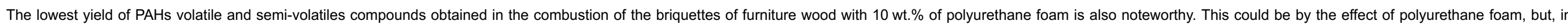
addition, some authors have found that densified fuels (briquettes or pellets) reduce light hydrocarbons, PAH emissions and particulate matters (PM) (Chen et al., 2004; Shao et al., 2016; Shen et al., 2012).

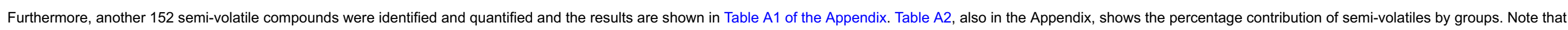
the combustion of briquettes of furniture wood and $10 \mathrm{wt} . \%$ of foam shows the highest percentage contribution of nitrogenated compounds.

The formation of PCDD/Fs and dioxin-like PCBs during the combustion of furniture waste has also been studied in the residential stove and in a laboratory scale reactor. 


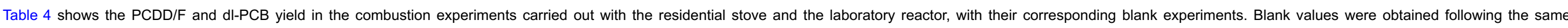

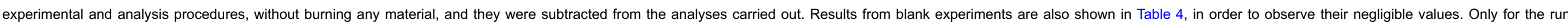
carried out with solid wood in the laboratory reactor, the blank is similar to the corresponding run, indicating that the PCDD/F and PCB formation was very small.

Table 4 Concentration of PCDD/Fs and dioxin-like PCBs $\left(\mathrm{ng} \mathrm{kg}^{-1}\right)$ in different samples and combustion experiments.

\begin{tabular}{|c|c|c|c|c|c|c|c|c|c|c|}
\hline & \multicolumn{6}{|c|}{ Gas from residential stove } & \multicolumn{4}{|c|}{ Gas from laboratory scale reactor } \\
\hline & $\begin{array}{l}\text { Furniture wood waste } \\
\text { (run I) }\end{array}$ & $\begin{array}{l}\text { Blank stove } \\
\quad \text { (run I) }\end{array}$ & $\begin{array}{l}\text { Furniture wood waste } \\
\text { (run II) }\end{array}$ & $\begin{array}{l}\text { Blank stove } \\
\text { (run II) }\end{array}$ & $\begin{array}{l}\text { Briquettes of furniture wood and } \\
10 \mathrm{wt} . \% \text { of foam }\end{array}$ & $\begin{array}{l}\text { Blank stove } \\
\text { (run III) }\end{array}$ & $\begin{array}{l}\text { Furniture wood waste } \\
\qquad(n=3)^{a}\end{array}$ & $\begin{array}{l}\text { Furn. wood } \\
\text { blank }\end{array}$ & $\begin{array}{l}\text { Solid } \\
\text { wood }\end{array}$ & $\begin{array}{l}\text { Solid wood } \\
\text { blank }\end{array}$ \\
\hline \multicolumn{11}{|l|}{ Operating conditions } \\
\hline Residence time (s) & $\sim 3.5$ & & $\sim 3.5$ & & $\sim 3.6$ & & $\sim 3.5$ & & $\sim 3.5$ & \\
\hline Furnace temperature $\left({ }^{\circ} \mathrm{C}\right)$ & $420-780$ & & $320-740$ & & $450-750$ & & 850 & & 850 & \\
\hline Ratio $\mathrm{CO} /\left(\mathrm{CO}_{2}+\mathrm{CO}\right)$ & 0.025 & & 0.035 & & 0.030 & & 0.070 & & 0.075 & \\
\hline \multicolumn{11}{|c|}{ PCDD/Fs and dioxin-like PCBs ( $\left.\mathrm{ng} \mathrm{kg}^{-1}\right)$} \\
\hline 2378-TCDF & 3.2 & 0.3 & 90 & 2.0 & 41 & 0.1 & $15.5 \pm 4.3$ & 0.2 & 1.5 & 0.1 \\
\hline 12378-PeCDF & 2.2 & 0.4 & 42 & 0.9 & 15 & 0.1 & $10.7 \pm 2.2$ & 1.0 & 1.4 & 0.7 \\
\hline 23478-PeCDF & 3.7 & 0.4 & 89 & 1.4 & 26 & 0.1 & $12.5 \pm 1.1$ & 0.9 & 1.4 & 0.6 \\
\hline 123478-HxCDF & 3.3 & 0.8 & 30 & 0.6 & 16 & 0.1 & $5.8 \pm 1.1$ & 1.1 & 1.1 & 0.7 \\
\hline 123678-HxCDF & 2.8 & 0.5 & 28 & 0.6 & 13 & 0.2 & $7.5 \pm 1.7$ & 1.0 & 0.9 & 0.7 \\
\hline 234678-HxCDF & 3.3 & 0.8 & 53 & 0.9 & 23 & nd & $11.5 \pm 7.9$ & 1.9 & 1.8 & 1.3 \\
\hline 123789-HxCDF & 2.0 & 0.8 & 6.7 & 0.2 & 4.2 & 0.1 & $3.4 \pm 4.6$ & 1.2 & 0.5 & 0.8 \\
\hline 1234678-HpCDF & 11.5 & 2.5 & 103 & 1.5 & 41 & 0.4 & $12.2 \pm 10.4$ & 4.0 & 1.1 & 2.7 \\
\hline 1234789-HpCDF & 8.0 & 0.9 & 8.2 & 0.6 & 4.9 & 0.2 & $3.5 \pm 2.3$ & 1.9 & nd & 1.3 \\
\hline OCDF & 29 & 2.9 & 30 & 0.5 & 20 & 0.1 & $8.7 \pm 8.8$ & 3.3 & 2.2 & 2.5 \\
\hline 2378-TCDD & 3.2 & 0.8 & 3.1 & 0.5 & 1.5 & nd & $3.9 \pm 1.8$ & 0.5 & 0.3 & 0.3 \\
\hline 12378-PeCDD & 12.4 & 0.5 & 10.3 & 0.5 & 4.6 & 0.3 & $5.6 \pm 1.1$ & 0.9 & 0.3 & 0.6 \\
\hline 123478-HxCDD & 11.6 & 0.4 & 11.4 & 0.6 & 3.5 & nd & $1.3 \pm 0.5$ & 0.7 & 1.0 & 0.5 \\
\hline 123678-HxCDD & 33 & 0.9 & 20 & 0.6 & 8.1 & 0.5 & $3.7 \pm 2.6$ & 0.5 & nd & 0.4 \\
\hline 123789-HxCDD & 30 & 0.8 & 15 & 0.8 & 8.1 & 0.3 & $3.4 \pm 1.8$ & 0.9 & 2.2 & 0.6 \\
\hline 1234678-HpCDD & 655 & 4.2 & 233 & 0.9 & 131 & 0.6 & $3.4 \pm 1.8$ & 1.8 & 2.1 & 1.2 \\
\hline OCDD & 2335 & 11.4 & 530 & 180 & 533 & 0.8 & $7.2 \pm 9.3$ & 2.9 & 5.2 & 2.0 \\
\hline Total WHO-TEQ PCDD/Fs & 33 & 2.1 & 70 & 2 & 28 & 0.5 & $19.0 \pm 2.9$ & 2.5 & 2.0 & 1.7 \\
\hline PCB-81 & 3.0 & 0.2 & 72 & 0.7 & 18 & nd & $9.1 \pm 7.4$ & nd & nd & nd \\
\hline РCB-77 & 11.0 & 0.8 & 505 & 9.9 & 83 & 1.5 & $73 \pm 69$ & 4.3 & 4.6 & 2.9 \\
\hline PCB-123 & 7.7 & 0.3 & 487 & 4.7 & 120 & 0.4 & $25 \pm 30$ & 1.5 & 2.1 & 1.0 \\
\hline
\end{tabular}




\begin{tabular}{|c|c|c|c|c|c|c|c|c|c|c|}
\hline PCB-118 & 63.6 & 2.2 & 4738 & 47 & 1276 & 5.9 & $101 \pm 114$ & 16.0 & 25.7 & 10.8 \\
\hline PCB-114 & 1.6 & 0.3 & 193 & 2.3 & 34 & 0.1 & $2.4 \pm 3.3$ & 0.5 & nd & nd \\
\hline PCB-105 & 25.9 & 1.2 & 2637 & 21 & 559 & 2.0 & $38 \pm 40$ & 7.2 & 10.3 & 4.9 \\
\hline PCB-126 & 2.2 & 0.3 & 36 & 1.6 & 14 & 0.4 & $14.4 \pm 9.1$ & 0.5 & 2.9 & 0.4 \\
\hline PCB-167 & 11.4 & 0.1 & 276 & 1.9 & 91 & 0.2 & $6.9 \pm 5.6$ & 1.1 & 0.1 & 0.1 \\
\hline PCB-156 & 11.2 & 0.4 & 442 & 4.0 & 159 & 0.4 & $11.7 \pm 4.3$ & 2.5 & 2.7 & 1.7 \\
\hline PCB-157 & 1.1 & 0.1 & 77 & 0.9 & 38 & nd & $7.2 \pm 5.2$ & 0.7 & 0.5 & 0.5 \\
\hline PCB-169 & 1.4 & nd & 7.4 & 0.2 & 4.5 & nd & $23 \pm 37$ & 0.6 & 4.6 & 0.4 \\
\hline PCB-189 & 2.9 & nd & 66 & 0.3 & 18 & 0.2 & $3.7 \pm 3.3$ & 0.6 & 1.4 & 0.4 \\
\hline Total WHO-TEQ PCBs & 0.3 & 0.03 & 4.2 & 0.1 & 1.6 & 0.05 & $2.2 \pm 0.9$ & 0.07 & 0.4 & 0.05 \\
\hline $\begin{array}{l}\text { Total WHO-TEQ } \\
\text { (PCDD/Fs + PCBs) }\end{array}$ & 33 & 2 & 74 & 2 & 29 & 0.6 & $21.1 \pm 2.1$ & 2.6 & 2.5 & 1.8 \\
\hline
\end{tabular}

n.d.: value less than the $L O D \sim 0.1 \mathrm{ng} / \mathrm{kg}$ sample.

a Triplicate experiment.

The total toxicity equivalence was calculated using the toxicity equivalency factor given by the World Health Organization (Van den Berg et al., 2006).

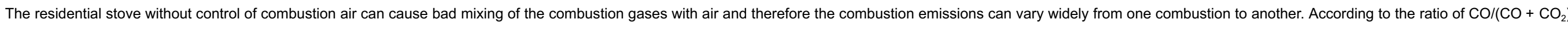

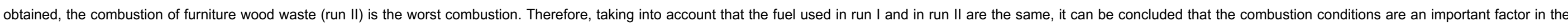

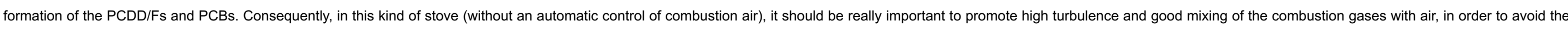
generation of incomplete combustion areas. In industrial boilers, operating with an excess of oxygen, good mixing will favour the destruction of organic pollutants.

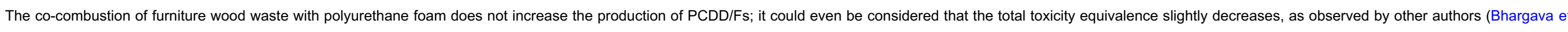

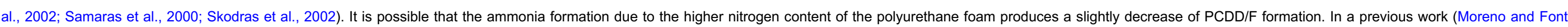

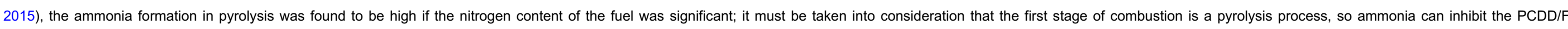
formation (Hajizadeh et al., 2012; Ruokojärvi et al., 2004, 1998). Other authors have also found ammonia emissions from biomass burning (Delmas et al., 1995; Goode et al., 2000; Yokelson et al., 2003).

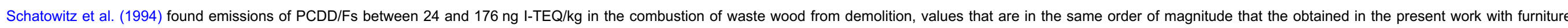
waste.

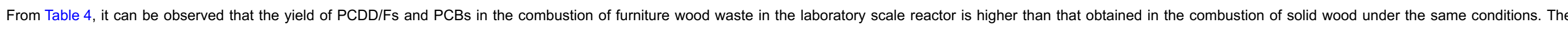

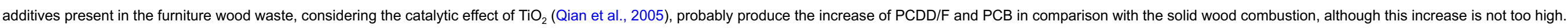

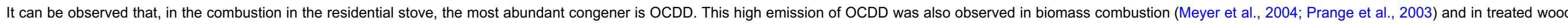
combustion (Salthammer et al., 1995; Wasson et al., 2005).

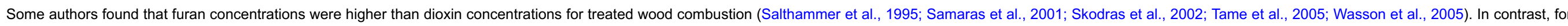

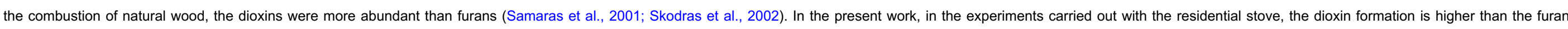

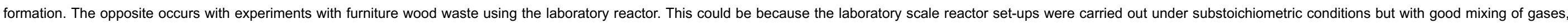
and therefore, a predominance of PCDFs occurred. Nevertheless, in the residential stove with an excess of air, but with bad mixing, PCDDs were the dominant compounds.

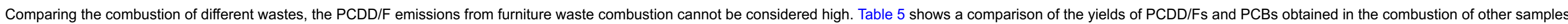


Table 5 Comparison of the emissions from combustion of different wastes.

\begin{tabular}{|c|c|c|c|}
\hline & Ratio $\mathrm{CO} /\left(\mathrm{CO}+\mathrm{CO}_{2}\right)$ & PCDD/Fs (ng TEQ/kg sample) & Reference \\
\hline \multicolumn{4}{|l|}{ Laboratory reactor at $850^{\circ} \mathrm{C}$} \\
\hline Furniture wood waste & 0.07 & 19 & Present work \\
\hline PVC & 0.09 & 4500 & \multirow[t]{5}{*}{ Conesa et al. (2009) } \\
\hline Sewage sludge with high metal content, mainly Fe & 0.11 & 1700 & \\
\hline Sewage sludge with low metal content & 0.08 & 55 & \\
\hline Waste lube oil & 0.46 & 80 & \\
\hline Polyester textiles & 0.11 & 14 & \\
\hline Pine needles & 0.11 & 49 & \multirow[t]{2}{*}{ Moltó et al. (2010) } \\
\hline Pine cones & 0.13 & 30 & \\
\hline \multicolumn{4}{|l|}{ Residential stove } \\
\hline Furniture waste & $0.03-0.04$ & $30-70$ & Present work \\
\hline Pine needles and cones & 0.12 & 175 & Moltó et al. (2010) \\
\hline
\end{tabular}

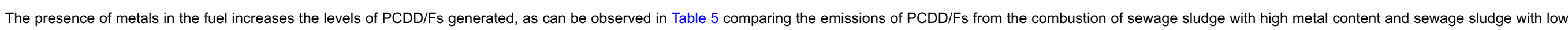

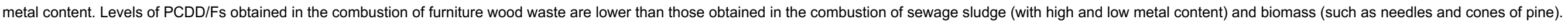

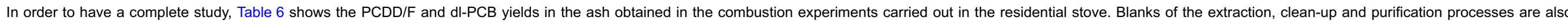
carried out and their values are also shown in Table 6.

Table 6 Concentration of PCDD/Fs and dioxin-like PCBs $\left(\mathrm{ng} \mathrm{kg}^{-1}\right)$ in the ashes from the residential stove combustions.

\begin{tabular}{|c|c|c|c|c|c|c|}
\hline & \multicolumn{6}{|c|}{ Ash from residential stove ( $\mathrm{gg} / \mathrm{kg}$ ash) } \\
\hline & Furniture wood waste (I) $(n=2)^{\mathrm{a}}$ & Blank (I) & Furniture wood waste (II) $(n=2)^{\mathrm{a}}$ & Blank (II) & Briquettes of furniture wood and $10 \mathrm{wt} . \%$ of foam $(n=2)^{\mathrm{a}}$ & Blank (III) \\
\hline Ratio $\mathrm{CO} /\left(\mathrm{CO}_{2}+\mathrm{CO}\right)$ & 0.025 & & 0.035 & & 0.030 & \\
\hline 2378-TCDF & $10.0 \pm 2.1$ & nd & $58.2 \pm 9.2$ & nd & $35.2 \pm 2.9$ & nd \\
\hline 12378-PeCDF & $5.6 \pm 0.9$ & 0.2 & $53.5 \pm 6.5$ & 0.1 & $26.9 \pm 2.5$ & 0.2 \\
\hline 23478-PeCDF & $7.3 \pm 0.6$ & 0.1 & $71.5 \pm 0.1$ & 0.1 & $36.9 \pm 2.7$ & 0.1 \\
\hline 123478-HxCDF & $4.2 \pm 0.6$ & 0.2 & $56.4 \pm 3.1$ & 0.1 & $24.9 \pm 1.8$ & 0.1 \\
\hline 123678-HxCDF & $4.1 \pm 0.5$ & 0.2 & $59.3 \pm 4.5$ & 0.1 & $26.1 \pm 0.9$ & 0.2 \\
\hline 234678-HxCDF & $7.4 \pm 0.8$ & 0.3 & $86.2 \pm 5.2$ & 0.2 & $41.7 \pm 4.9$ & 0.2 \\
\hline 123789-HxCDF & $1.2 \pm 0.8$ & 0.3 & $19.3 \pm 1.1$ & 0.2 & $8.4 \pm 0.1$ & 0.3 \\
\hline 1234678-HpCDF & $18.1 \pm 1.6$ & 0.7 & $175 \pm 29$ & 0.5 & $100 \pm 14$ & 0.6 \\
\hline
\end{tabular}




\begin{tabular}{|c|c|c|c|c|c|c|}
\hline 1234789-HpCDF & $1.6 \pm 0.02$ & 0.2 & $17.0 \pm 1.5$ & 0.2 & $6.5 \pm 0.5$ & 0.2 \\
\hline OCDF & $3.4 \pm 1.1$ & 0.7 & $50.7 \pm 3.3$ & 0.5 & $16.7 \pm 1.8$ & 0.6 \\
\hline 2378-TCDD & $0.4 \pm 0.1$ & 0.1 & $2.7 \pm 0.2$ & 0.1 & $1.2 \pm 0.1$ & 0.1 \\
\hline 12378-PeCDD & $2.1 \pm 0.5$ & 0.2 & $11.3 \pm 1.3$ & 0.1 & $4.7 \pm 0.6$ & 0.2 \\
\hline 123478-HxCDD & $1.1 \pm 0.1$ & 0.1 & $5.2 \pm 0.5$ & 0.1 & $2.1 \pm 0.3$ & 0.1 \\
\hline 123678-HxCDD & $5.9 \pm 0.02$ & 0.3 & $8.6 \pm 2.0$ & 0.2 & $4.2 \pm 0.7$ & 0.2 \\
\hline 123789-HxCDD & $4.0 \pm 0.5$ & 0.3 & $7.3 \pm 0.7$ & 0.2 & $3.7 \pm 0.5$ & 0.2 \\
\hline 1234678-HpCDD & $32.8 \pm 1.3$ & 0.4 & $57.2 \pm 13.8$ & 0.3 & $32 \pm 6$ & 0.4 \\
\hline OCDD & $111 \pm 5$ & 0.7 & $79.5 \pm 6.9$ & 0.5 & $64 \pm 10$ & 0.6 \\
\hline Total WHO-TEQ PCDD/Fs & $9.2 \pm 0.3$ & 0.5 & $70 \pm 3$ & 0.3 & $33.9 \pm 0.2$ & 0.4 \\
\hline PCB-81 & $2.4 \pm 0.2$ & nd & $37 \pm 0.1$ & nd & $22.3 \pm 2.6$ & nd \\
\hline РCB-77 & $22.1 \pm 4$ & 0.7 & $181 \pm 7$ & 0.5 & $85.0 \pm 3.2$ & 0.6 \\
\hline PCB-123 & $2.7 \pm 0.7$ & 0.3 & $232 \pm 47$ & 0.2 & $109 \pm 17$ & 0.3 \\
\hline PCB-118 & $13.6 \pm 2.8$ & 3.1 & $2599 \pm 571$ & 2.1 & $1213 \pm 149$ & 2.6 \\
\hline PCB-114 & $0.4 \pm 0.3$ & nd & $71 \pm 16$ & nd & $34 \pm 5$ & 0.1 \\
\hline PCB-105 & $8.2 \pm 2.1$ & 1.3 & $1277 \pm 298$ & 0.9 & $541 \pm 67$ & 1.1 \\
\hline PCB-126 & $5.8 \pm 1.1$ & 0.3 & $80 \pm 4$ & 0.2 & $28.8 \pm 1.9$ & 0.2 \\
\hline PCB-167 & $3.2 \pm 1.6$ & 0.2 & $112 \pm 25$ & 0.1 & $47.0 \pm 7.7$ & 0.2 \\
\hline PCB-156 & $15.9 \pm 18.1$ & 0.2 & $282 \pm 62$ & 0.2 & $97.5 \pm 12.5$ & 0.2 \\
\hline PCB-157 & $12.0 \pm 15.3$ & nd & $86 \pm 17$ & nd & $31.8 \pm 5.7$ & nd \\
\hline PCB-169 & $1.5 \pm 0.2$ & nd & $19 \pm 1$ & nd & $7.8 \pm 1.8$ & nd \\
\hline PCB-189 & $1.4 \pm 0.1$ & 0.6 & $27 \pm 3$ & 0.4 & $10.3 \pm 2.1$ & 0.5 \\
\hline Total WHO-TEQ PCBS & $0.6 \pm 0.1$ & 0.03 & $9 \pm 0.5$ & 0.02 & $3 \pm 0.3$ & 0.02 \\
\hline Total WHO-TEQ (PCDD/Fs + PCBs) & $10 \pm 0.4$ & 0.5 & $78 \pm 2$ & 0.4 & $37 \pm 0.4$ & 0.4 \\
\hline
\end{tabular}

n.d.: value less than the $L O D \sim 0.1 \mathrm{ng} / \mathrm{kg}$ sample.

a Duplicate analysis.

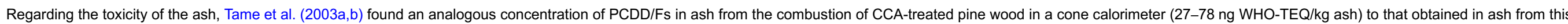

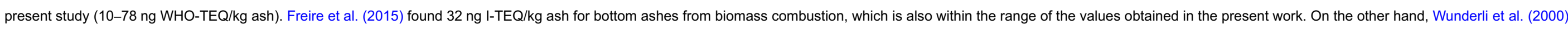
found low concentrations of PCDD/Fs in ash (4-11 ng I-TEQ/kg ash), in urban waste wood bottom ashes from incinerators and wood combustion facilities in Switzerland.

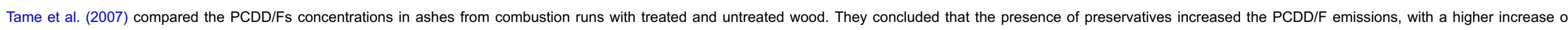
furans.

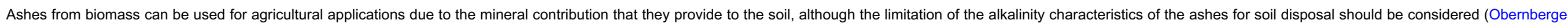
and Supancic, 2009; Pitman, 2006; van Loo and Koppejan, 2008). 


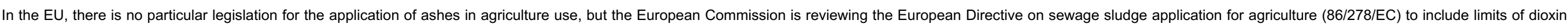

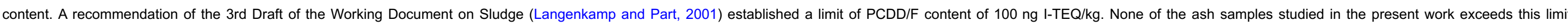
proposed.

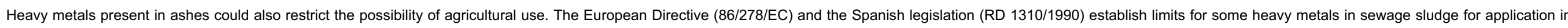
agriculture (Cd: 20, Cu: 1000, Ni: 300, Pb: 750, Zn:2500, Hg: 16, Cr: 1000, in mg/kg).

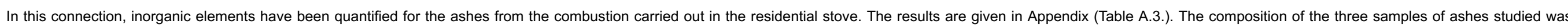
quite similar. Ash samples do not exceed the heavy metal limits of the legislation, except for the $\mathrm{Pb}$ content in the ash from the 2nd experiment of combustion of furniture wood waste that is $1300 \mathrm{mg} / \mathrm{kg}$ ash.

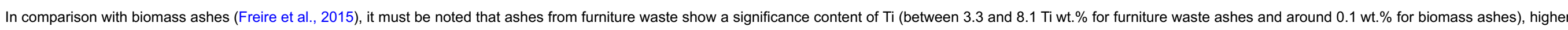

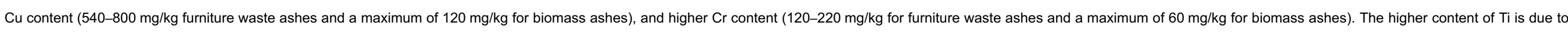

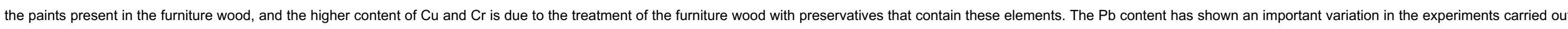
in the present work (between 20 and $1300 \mathrm{mg} / \mathrm{kg}$ ash); the maximum Pb content found in biomass ash by Freire et al. (2015) was $100 \mathrm{mg} / \mathrm{kg}$ ash.

\section{Conclusions}

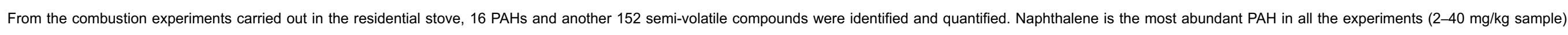

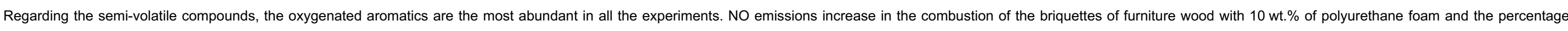

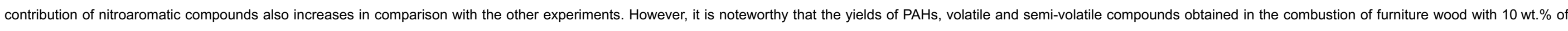
polyurethane foam are the lowest. This fact could be explained by the composition of the fuel and also by the densification of the fuel.

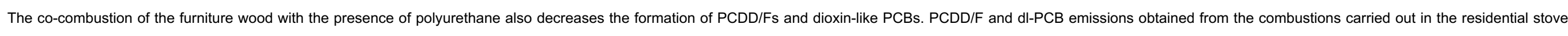

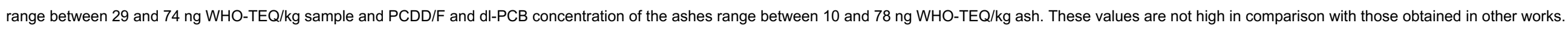

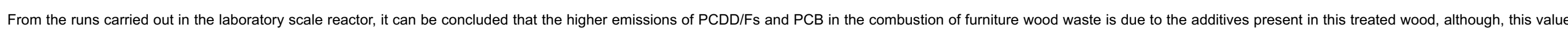

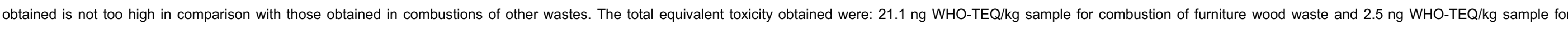
combustion of solid wood.

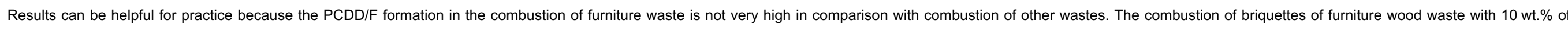

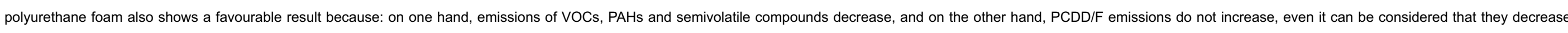

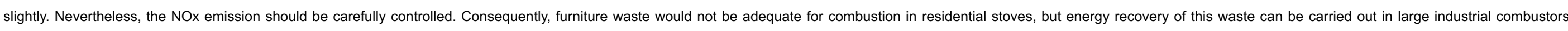
without special problems due to the volatiles evolved.

\section{Uncited reference}

Fontetal. (2003).

\section{Acknowledgment}

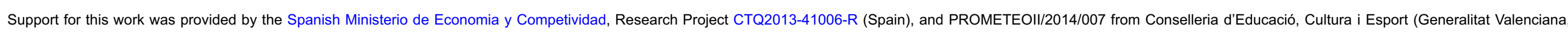
Spain). Authors also acknowledge Univ. of Alicante for financial support through UAUSTI15-04.

\section{Appendix A. Supplementary material}

Supplementary data associated with this article can be found, in the online version, at http://dx.doi.org/10.1016/j.wasman.2016.09.046. 


\section{References}

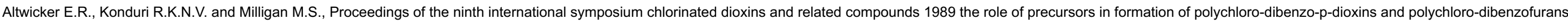
during heterogeneous combustion, Chemosphere 20, 1990, 1935-1944.

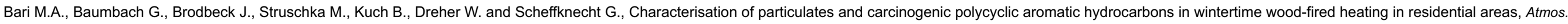
Environ. 45, 2011, 7627-7634

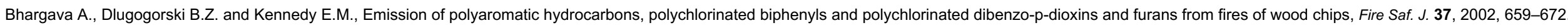

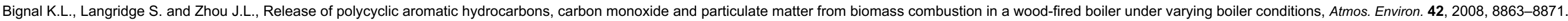
BMU, 2002. Ordinance on the Management of Waste Wood, dated 15 August 2002. Bundesministerium für Umwelt, Naturschutz, Bau und Reaktorsicherheit (BMU).

CEN, 2010. CEN-TS 14961-1:2010. Technical Specification: Solid Biofuels. Fuel Specifications and Clases. Part 3: Wood Briquettes for Non-industrial Use.

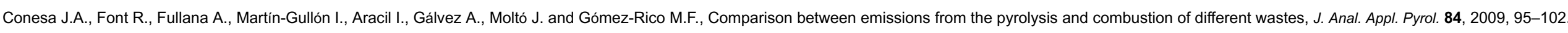

Chen Y., Bi X., Mai B., Sheng G. and Fu J., Emission characterization of particulate/gaseous phases and size association for polycyclic aromatic hydrocarbons from residential coal combustion, Fuel 83, $2004,781-790$.

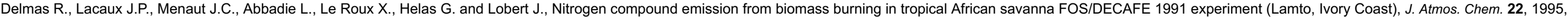
175-193.

Di Blasi C., Branca C. and Galgano A., Thermal and catalytic decomposition of wood impregnated with sulfur- and phosphorus-containing ammonium salts, Polym. Degrad. Stab. 93, 2008, 335-346.

EPA, U.S. Environmental Protection Agengcy. Method 3545A, 2007, Pressurized Fluid Extraction (PFE); Washington, D.C..

EPA, 2014. U.S. Environmental Protection Agency. Method 8270D. Semivolatile Organic Compounds by Gas Chromatography/Mass Spectrometry (GC/MS). Washington, D.C.

Evtyugina M., Alves C., Calvo A., Nunes T., Tarelho L., Duarte M., Prozil S.O., Evtuguin D.V. and Pio C., VOC emissions from residential combustion of Southern and mid-European woods, Atmos. Environ. 83, 2014, 90-98.

Font R., Esperanza M. and Nuria Garcia A., Toxic by-products from the combustion of Kraft lignin, Chemosphere 52, 2003, 1047-1058.

Freire M., Lopes H. and Tarelho L.A.C., Critical aspects of biomass ashes utilization in soils: composition, leachability, PAH and PCDD/F, Waste Manage. 46, 2015, 304-315.

Gao M., Pan D.X. and Sun C.Y., Study on the thermal degradation of wood treated with amino resin and amino resin modified with phosphoric acid, J. Fire Sci. 21, $2003,189-201$.

Garrido M.A. and Font R., Pyrolysis and combustion study of flexible polyurethane foam, J. Anal. Appl. Pyrol. 113, 2015, 202-215.

Gómez-Rico M.F. and Font R.A.F., Study of PCDD/Fs formation during composting of sewage sludge in different conditions, Organohalogen Compd. 69, $2007,1570-1573$.

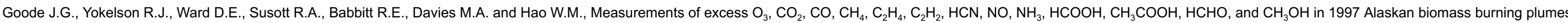

by airborne Fourier transform infrared spectroscopy (AFTIR), J. Geophys. Res. Atmos. 105, 2000, 22147-22166.

Hajizadeh Y., Onwudili J.A. and Williams P.T., Effects of gaseous $\mathrm{NH}_{3}$ and $\mathrm{SO}_{2}$ on the concentration profiles of PCDD/F in flyash under post-combustion zone conditions, Waste Manage. 32, 2012, 1378-1386.

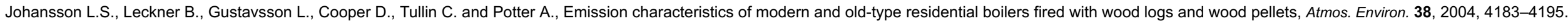

Khalfi A., Trouvé G., Delobel R. and Delfosse L., Correlation of CO and PAH emissions during laboratory-scale incineration of wood waste furnitures, J. Anal. Appl. Pyrol. 56, $2000,243-262$.

Kozlowski R. and Wladyka-Przybylak M., 9 - Natural polymers, wood and lignocellulosic materials, In: Horrocks A.R. and Price D., (Eds.), Fire Retardant Materials, 2001 , Woodhead Publishing, $293-317$.

Langenkamp H. and Part P., Organic Contaminants in Sewage Sludge for Agricultural Use, 2001, European Commision. 
Lavric E.D., Konnov A.A. and Ruyck J.D., Dioxin levels in wood combustion-a review, Biomass Bioenergy 26, 2004, 115-145.

McDonald J.D., Zielinska B., Fujita E.M., Sagebiel J.C., Chow J.C. and Watson J.G., Fine particle and gaseous emission rates from residential wood combustion, Environ. Sci. Technol. 34, $2000,2080-2091$.

McKay G., Dioxin characterisation, formation and minimisation during municipal solid waste (MSW) incineration: review, Chem. Eng. J. 86, 2002, 343-368.

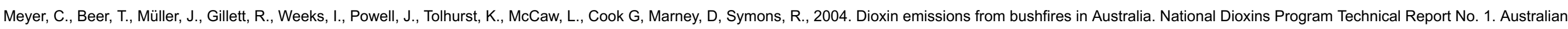
Government Department of the Environment and Heritage, Canberra.

Moltó J., Font R. and Conesa J.A., Study of the organic compounds produced in the pyrolysis and combustion of used polyester fabrics, Energy Fuels 20, 2006, 1951-1958.

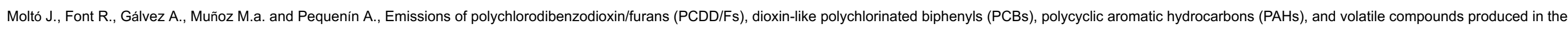
combustion of pine needles and cones, Energy Fuels 24, 2010, 1030-1036.

Moreno A.I. and Font R., Pyrolysis of furniture wood waste: decomposition and gases evolved, J. Anal. Appl. Pyrol. 113, 2015, 464-473.

Moreno A.I., Font R. and Conesa J.A., Physical and chemical evaluation of furniture waste briquettes, Waste Manage. 49, 2016, $245-252$.

Muñoz M., Font R., Gómez-Rico M.F. and Moreno A.I., Chlorophenols and PCDD/Fs during sewage sludge composting, Organohalogen Compd. 72, 2010, 1362-1365.

Muñoz M., Gomez-Rico M.F. and Font R., PCDD/F formation from chlorophenols by lignin and manganese peroxidases, Chemosphere 110, 2014, 129-135.

Obernberger I., Biedermann F., Widmann W. and Riedl R., Concentrations of inorganic elements in biomass fuels and recovery in the different ash fractions, Biomass Bioenergy 12, 1997, 211-224.

Obernberger, I., Supancic, K., 2009. Possibilities of ash utilisation from biomass combustion plants. In: Energies, E.-R. (Ed.), 17th European Biomass Conference \& Exhibition, Hamburg.

Pandelova M., Lenoir D. and Schramm K.W., Inhibition of PCDD/F and PCB formation in co-combustion, J. Hazard. Mater. 149, 2007, 615-618.

Pitman R.M., Wood ash use in forestry - a review of the environmental impacts, Forestry 79, 2006, 563-588.

Pizzi A. and Mittal K.L., Wood Adhesives, 2011, CRC Press; VSP.

Prange J.A., Gaus C., Weber R., Päpke O. and Müller J.F., Assessing forest fire as a potential PCDD/F source in Queensland, Australia, Environ. Sci. Technol. 37, 2003, 4325-4329.

Preto F., McCleave R., McLaughlin D. and Wang J., Dioxins/furans emissions from fluidized bed combustion of salt-laden hog fuel, Chemosphere 58, $2005,935-941$.

Qian Y., Zheng M., Liu W., Ma X. and Zhang B., Influence of metal oxides on PCDD/Fs formation from pentachlorophenol, Chemosphere 60, 2005, 951-958.

Reisen F., Bhujel M. and Leonard J., Particle and volatile organic emissions from the combustion of a range of building and furnishing materials using a cone calorimeter, Fire Saf. J. 69, $2014,76-88$.

Roy M.M. and Corscadden K.W., An experimental study of combustion and emissions of biomass briquettes in a domestic wood stove, Appl. Energy 99, $2012,206-212$.

Ruokojärvi P.H., Asikainen A.H., Tuppurainen K.A. and Ruuskanen J., Chemical inhibition of PCDD/F formation in incineration processes, Sci. Total Environ. 325, $2004,83-94$.

Ruokojärvi P.H., Halonen I.A., Tuppurainen K.A., Tarhanen J. and Ruuskanen J., Effect of gaseous inhibitors on PCDD/F Formation, Environ. Sci. Technol. 32, 1998, 3099-3103.

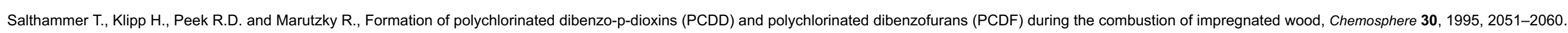

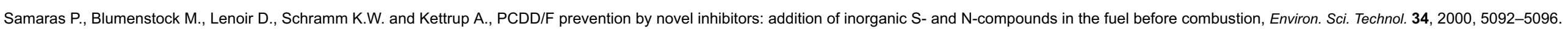

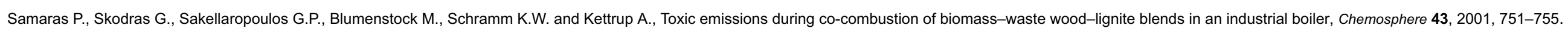

Schatowitz B., Brandt G., Gafner F., Schlumpf E., Bühler R., Hasler P. and Nussbaumer T., Dioxin emissions from wood combustion, Chemosphere 29, 1994, 2005-2013. 


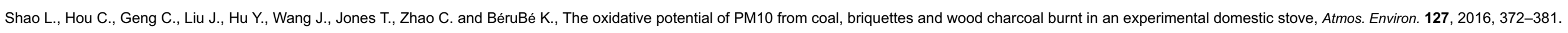

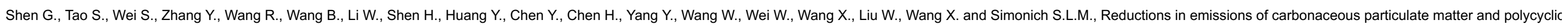
aromatic hydrocarbons from combustion of biomass pellets in comparison with raw fuel burning, Environ. Sci. Technol. 46, 2012, 6409-6416.

Skodras G., Grammelis P., Samaras P., Vourliotis P., Kakaras E. and Sakellaropoulos G.P., Emissions monitoring during coal waste wood co-combustion in an industrial steam boiler, Fuel 81, $2002,547-554$.

Stubenberger G., Scharler R., Zahirović S. and Obernberger I., Experimental investigation of nitrogen species release from different solid biomass fuels as a basis for release models, Fuel 87, 2008, 793-806.

Tame N.W., Dlugogorski B.Z. and Kennedy E.M., Assessing influence of experimental parameters on formation of PCDD/F from ash derived from fires of CCA-treated wood, Environ. Sci. Technol. 37, 2003a, 4148-4156.

Tame N.W., Dlugogorski B.Z. and Kennedy E.M., Increased PCDD/F formation in the bottom ash from fires of CCA-treated wood, Chemosphere 50, 2003b, 1261-1263.

Tame N.W., Dlugogorski B.Z. and Kennedy E.M., PCDD/F formation in flaming combustion, smoldering, and oxidative pyrolysis of 'eco-friendly' treated wood, Proc. Combust. Inst. 30, 2005, 1237-1243.

Tame N.W., Dlugogorski B.Z. and Kennedy E.M., Formation of dioxins and furans during combustion of treated wood, Prog. Energy Combust. Sci. 33, 2007, 384-408.

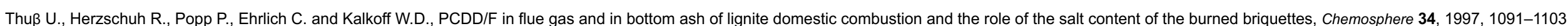

Tissari J., Hytönen K., Lyyränen J. and Jokiniemi J., A novel field measurement method for determining fine particle and gas emissions from residential wood combustion, Atmos. Environ. 41, $2007,8330-8344$.

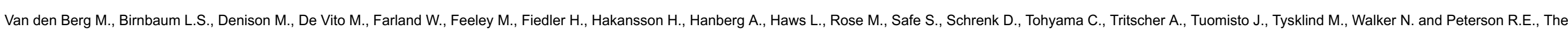
2005 World Health Organization reevaluation of human and mammalian toxic equivalency factors for dioxins and dioxin-like compounds, Toxicol. Sci. 93, 2006, 223-241.

van Loo S. and Koppejan J., The Handbook of Biomass Combustion and Co-Firing, 2008, Earthscan; London.

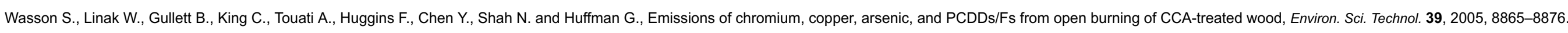

Werther J., Saenger M., Hartge E.U., Ogada T. and Siagi Z., Combustion of agricultural residues, Prog. Energy Combust. Sci. 26, 2000, 1-27.

Williams A., Jones J.M., Ma L. and Pourkashanian M., Pollutants from the combustion of solid biomass fuels, Prog. Energy Combust. Sci. 38, 2012, $113-137$.

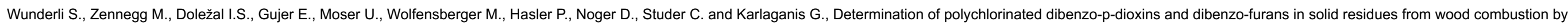
HRGC/HRMS, Chemosphere 40, 2000, 641-649.

Yasuhara A., Katami T. and Shibamoto T., Formation of PCDDs, PCDFs, and coplanar PCBs from incineration of various woods in the presence of chlorides, Environ. Sci. Technol. 37, 2003, 1563-1567.

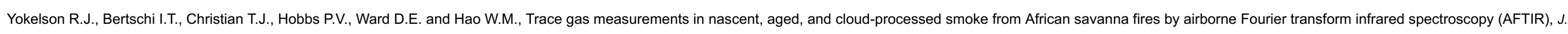
Geophys. Res. Atmos. 108, 2003, n/a-n/a.

\section{Appendix A. Supplementary material}

Multimedia Component 1

Supplementary data 1

Graphical abstract 


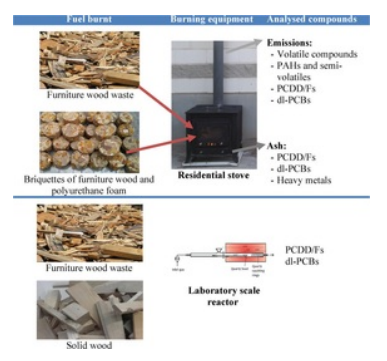

Highlights

- Co-combustion with polyurethane foam can be carried out without toxic risk.

- More than 150 semi-volatile compounds, PCDD/Fs and dl-PCBs evolved have been quantified.

- PCDD/F + PCB emissions are low (29-74 ng WHO-TEQ/kg) in comparison with biomass combustions.

\section{Queries and Answers}

Query: Please provide a definition for the significance of bold, italics in Tables 3, 4 and 6 .

Answer: Italics and bold letters are used to distingish the different parts of the tables. Although it could be without the use of italics or bold.

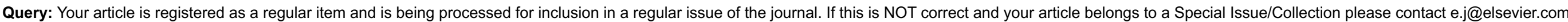
immediately prior to returning your corrections.

Answer: It does not belong to a Special Issue

Query: The author names have been tagged as given names and surnames (surnames are highlighted in teal color). Please confirm if they have been identified correctly.

Answer: Yes, they are identified correctly.

Query: The country name has been inserted for affiliation. Please check, and correct if necessary.

Answer: It is correct

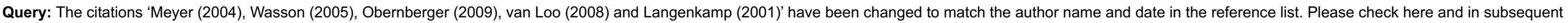
occurrences, and correct if necessary.

Answer: Yes

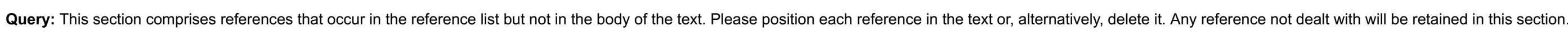
Answer: This reference is positioned now in the text. 